\title{
The Study of the Springback Control Algorithm Based on Improved Fast Fourier Transform
}

\author{
Wenjuan Liu, a, Qinghua Zhou ${ }^{2, b}$ and Huizhang Zhang ${ }^{1, c}$ \\ ${ }^{1}$ School of Computer Science, Zhaoqing University, Zhaoqing 526061, China. \\ ${ }^{2}$ School of Mathematics and Statistics, Zhaoqing University, Zhaoqing 526061, China. \\ a77047605@qq.com, bzqhmath@126.com, ${ }^{\mathrm{c}} 1121031894 @ q q . c o m$
}

Keywords: Fast Fourier Transform, Springback control, wavelet analysis.

\begin{abstract}
This paper proposed a mould modification algorithm based on improved Fast Fourier Transform. The wavelet transform and Fourier transform are used to solve the shape transfer function of the springback closed-loop control system. Through the shape transfer function to predict the mould surface shape corresponding to the stamping parts shape in the same process conditions, so as to achieve the purpose of correcting the mould surface to compensate springback error. Finally, this paper verified the effectiveness of mould surface springback correction algorithm based on multi-curvature stamping experiment.
\end{abstract}

\section{Introduction}

In recent years, many scholars have adopted numerical simulation technology study on stamping springback compensation, it is the premise that springback simulation accuracy must be guaranteed, and this is the defect of the numerical simulation. In addition, there are some scholars have adopted mould experiment measurement correction method to control the springback. C.Hindman of the Arizona university [1] and Dr R.D.Webb of Massachusetts institute of technology[2] used Fourier transform to describe the surface shape of the stamping mould and stamping parts,to implement the compensation of stamping springback.

This paper proposes a springback closed loop control system model based on fast Fourier transform. Under the same process condition, the frequency domain transfer relationship between mould and the corresponding stamping parts were obtained, and then predict the correction position and correction quantity size of mould springback compensation, the springback compensation of stamping parts is realized. Finally, using multi-curvature component stamping experiments verify the effectiveness of springback compensation algorithm. The method describes surface geometry of the mould and the corresponding stamping parts based on improved fast Fourier transform.

\section{Springback closed loop control system model}

Sheet metal stamping springback compensation system can be abstracted into a mould correction closed loop control system.Fourier transform is used to establish bridges from time domain to frequency domain, and Fourier inverse transform is used to establish bridges from frequency domain to time domain transformation of discrete quantity. Using Fourier transform can achieve the purpose of processing surface discrete data, and using Fourier inverse transform can get the desires of mould surface, which is the basic principle of using Fourier transform to mould modification. In the frequency domain the transform function can be expressed as

$$
H(d)=\frac{F_{2}(\Delta d)}{F_{2}(\Delta p)}
$$

In the formula, $\mathrm{H}$ for mould stamping parts deviation frequency domain transfer function, $F_{2}(\bullet)$ for two-dimensional fast Fourier transform, that is, FFT transformation.

Formula (1) show that in the stamping process parameters constant, the small changes of stamping mould shape $\Delta d$ is proportional to the changes of the corresponding stamping parts shape $\Delta p$ after two-dimensional FFT transform in frequency domain, scale factor is $\mathrm{H}$. 
Suppose $\mathrm{p}^{*}$ is the ideal shape of the product, $\mathrm{d}^{*}$ for the mould surface of the mould design. Under the constant process parameters, the function relation between $\mathrm{Di}$ and $\mathrm{Pi}$ can be the same. And stamping deviation frequency domain transfer function $\mathrm{H}$ reflects such function relation, the function $\mathrm{H}(\mathrm{d})$ is as follows:

$$
H(d)=\frac{F_{2}(\Delta d)}{F_{2}(\Delta p)}=\frac{F_{2}\left(d^{*}\right)-F_{2}\left(d_{1}\right)}{F_{2}\left(p^{*}\right)-F_{2}\left(p_{1}\right)}
$$

$F_{2}\left(d^{*}\right)$ inverse FFT transform can get a set of discrete data points of the ideal mould surface.

$$
d^{*}=F_{2}^{-1}\left[F_{2}\left(d^{*}\right)\right]
$$

\section{Improved Fourier Transform Algorithm}

The mould profile correction transfer function describes the mould surface to the stamping parts surface function, There is representation error problem with the Fourier handle this semaphore. So this article in the time domain to frequency domain transformation using a wavelet transform based on Fourier transform, wavelet transform can reflect the nature of the signal from the time domain to frequency domain, but also can reflect the nature of the signal in time domain of instant changes, so as to make up for the defect of previous discrete Fourier transform in the spatial domain, can make the closed-loop control system is more close to the optimal solution[3, 4]. Through the analysis found that: if the wavelet function is taken as a function of Shannon wavelet type, the fast Fourier transform may be used to complete the wavelet transform[5, 6], and by means of fast Fourier transform can realize wavelet transform。

For a discrete signal $\mathrm{f}(\mathrm{n}), \mathrm{n}=0,1,2$, 、 、, $\mathrm{N}-1 ; \mathrm{N}=2^{l}, l \in Z$, Sets the FFT of $\mathrm{f}(\mathrm{n})$ is

$$
F(k)=(1 / N) \sum_{n=0}^{N-1} f(n) \exp \left(\left(-j 2 n k^{\pi}\right) / N\right)
$$

Fourier transform of $\mathrm{F}(\mathrm{k})$ are as follows:

$$
F F(m)=(1 / N) \sum_{m=0}^{N-1} f(k) \exp \left(-j 2 m k^{\pi} / N\right), \mathrm{m}=0, \quad 1, \cdots, \quad \mathrm{N}-1
$$

Take a non-compact supported orthogonal wavelet, $f(x) \in L^{2}(R)$, then

$$
w_{f}(\lambda, x)=\frac{\sqrt{\lambda}}{2 \pi}\left[\int_{2 \pi / \lambda}^{\pi / \lambda} F(w) \exp (-j x w) d w+\int_{\pi / \lambda}^{2 \pi / \lambda} F(w) \exp (-j x w) d w\right]
$$

By the formula (5) to give:

$$
\begin{gathered}
w_{f}\left(2^{-i}, 2^{-i} m\right)=\left[2 /\left(2^{i} \sqrt{2^{i}}\right)\right] F F_{i}(m) \\
F F_{i}(m)=\left(1 / N_{i}\right) \sum_{k=0}^{N_{i}-1} F_{i}(k) \exp \left(-j 2 m k^{\pi} / N_{i}\right)
\end{gathered}
$$

So, by the formula (8) will transform wavelet signal into constant times of the signal's Fourier transform, which can use FFT to discrete orthogonal wavelet transform and inverse transformation.

\section{Identification Algorithm MATLAB realization}

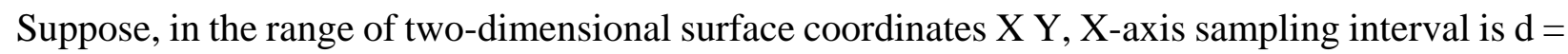
$\mathrm{D} / \mathrm{L}$, where $\mathrm{L}$ is the number of sampling points, $\mathrm{D}$ is the width of the surface. On the $\mathrm{Y}$-axis $\mathrm{L}$ sample points taken to produce a matrix.The realization steps of Identification Algorithm MATLAB are as follows:

(1) Matrix is transformed into a column vector using reshape function, as a discrete time-domain representation, then DFT matrix is generated by function $\mathrm{Mn}=\mathrm{dftmtx}($ length(x)). The matrix multiplication, $\mathrm{Fx}=\mathrm{Mn} * \mathrm{x}$ is equivalent to the DFT. Thus calculate the Fourier transform Fx ( $\mathrm{R}, \theta$ )of the $\theta$ direction projection set, $\mathrm{R}$ represents different mould surface and stamping parts surface shape 
in time domain data array. Then find a different $\mathrm{R}$ values $(\mathrm{R}, \theta)$, to give the mould surface and the stamping-type surface shape data Fourier frequency domain representation.

(2) According to the algorithm, based on the Fourier frequency domain representation of the mould surface and stamping parts surface shape data, seeking the wavelet frequency domain representation of the mould surface and the stamping parts surface shape data. Calculate $F x_{i}(K)\left(i=0,1, \ldots, N_{i}-1\right)$ first, using the above Fourier frequency domain representation method calculating the $\mathrm{FFx}_{i}(m)(m=0,1, \ldots, N-1)$. Equivalent to twice Fourier transform to the original mould surface and stamping molding surface shape data.

(3) Calculate the wavelet transform of the mould surface and the stamping parts surface shape data $w_{f}\left(2^{-i}, 2^{-i} m\right)=\left[2 /\left(2^{i} \sqrt{2^{i}}\right)\right] F F_{i}(m)$, taken $\mathrm{i}=2$, calculate $w_{f}\left(2^{-i}, 2^{-i} m\right)\left(m=0,1, \ldots, N_{i}-1\right)$ under all scales $2^{-i}(i=1,2, \ldots, j)$, can get the multi-scale decomposition $C^{-i}, D^{-j}, D^{-j+1}, \ldots, D^{-1}$ of the signal $\mathrm{f}(\mathrm{n})$.

(4) By transfer function identification process, depending on different types of surface $\mathrm{f}(\mathrm{n})$ multi scale shape data decomposition to calculate the transfer function of the closed loop control system, finally obtained the frequency domain representation of the mould surface according to the transfer function.

(5) According to the above represents the inverse process of obtaining the required fields of the mould surface.

\section{Verification of mould surface springback correction algorithm}

Based on multi-curvature stamping experiment, this paper verified the effectiveness of mould surface springback correction algorithm in the mould surface correction closed loop control system, designed and manufactured three different curvature surface mould. Experiment material select 150 $\mathrm{mm} \times 50 \mathrm{~mm}$, 08AL steel plate with $0.8 \mathrm{~mm}$ thickness, forming pressure is 70KN.

The representation of a time domain to frequency domain of multi-curvature Mould surface and the multi-curvature stamping parts, as shown in figure 1 , figure 2.

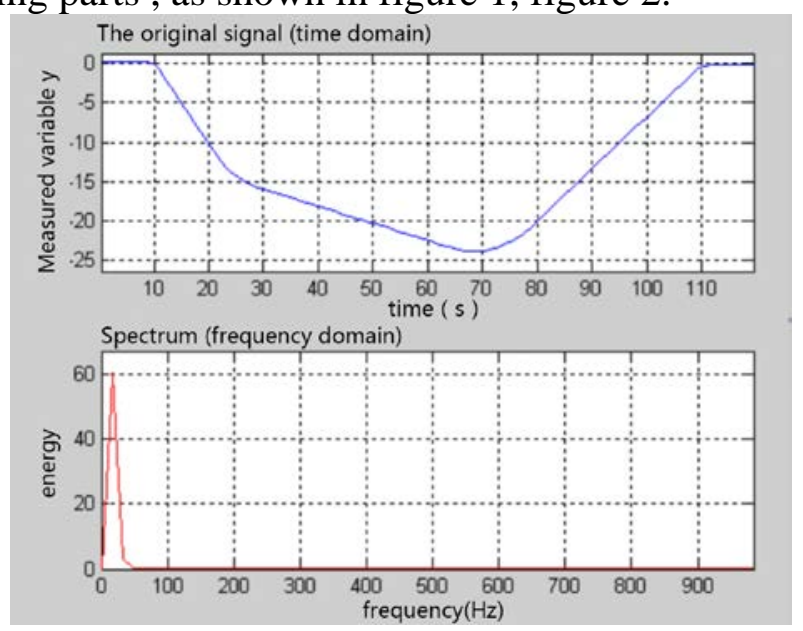

Fig.1 Mould1 surface time-frequency domain representation 


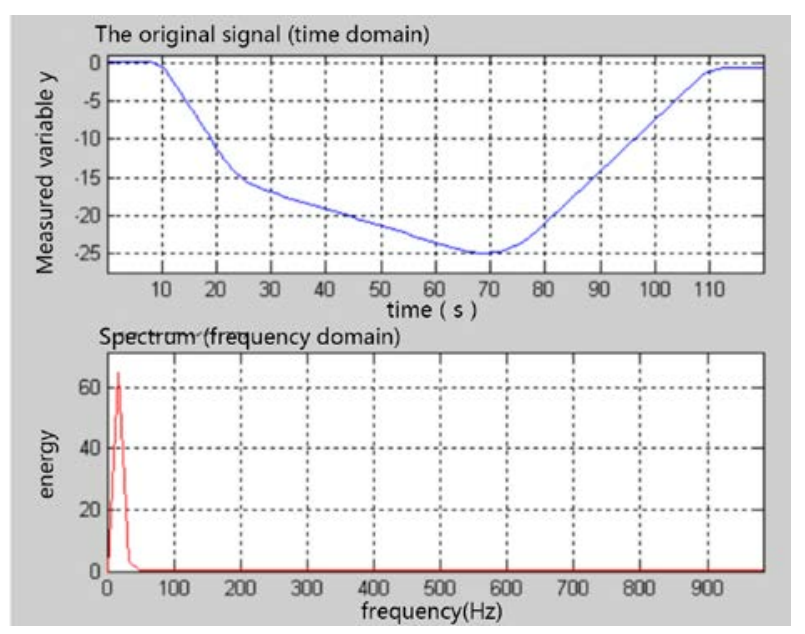

Fig.2 Stamping parts1 surface time-frequency domain representation

The comparison of the mould surface profile correction results and the actual mould surface profile is shown in figure 3, mould profile correction algorithm error distribution curves is shown in figure 4,as can be seen from the figure, the error of mould surface correction algorithm is $0.22 \mathrm{~mm}$ or less , the largest error is $0.219 \mathrm{~mm}$, the average error is $0.089 \mathrm{~mm}$. The results show that the mould correction compensation algorithm of this paper puts forward, can correctly predict the mould surface shape corresponding to the stamping parts shape in the same process conditions, so as to achieve the purpose of correcting the mould surface to compensate springback error.

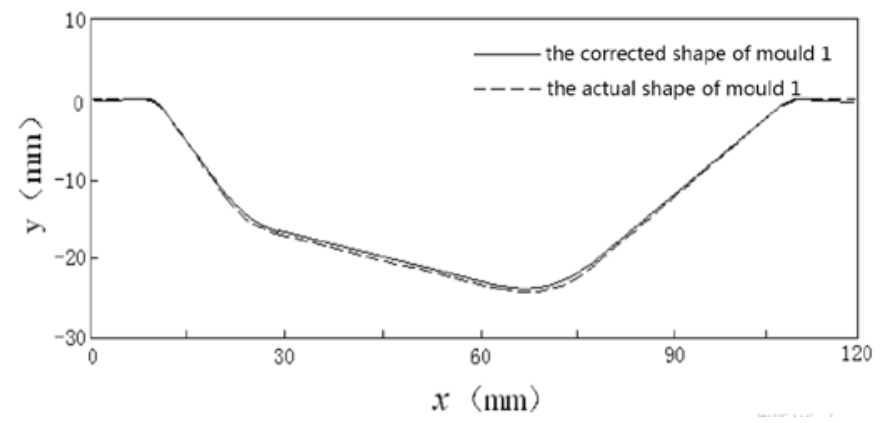

Fig.3 The comparison of Mould surface correction results

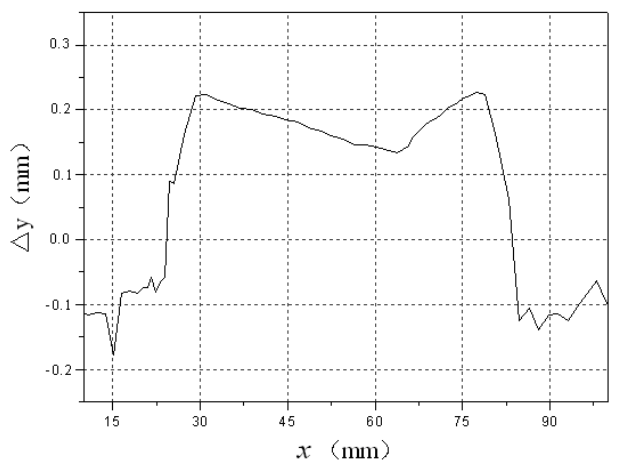

Fig.4 Mould profile correction algorithm error distribution curves

\section{Summary}

This paper proposed a mould modification algorithm based on frequency transfer function. The frequency domain representation of tools and parts surface discrete data is first investigated using wavelet transform. The wavelet transform and Fourier transform are then used to solve the shape transfer function of the closed-loop control system. The springback error is effectively controlled by the modification of mould surface. The key problems of the time-frequency domain representation of tools and parts surface discrete data, the solution of shape transfer function of the closed-loop control 
system, compentation of springback error is solved. Moreover, the modification algorithm has practical guiding values for die precision design and springback compensation.

\section{Acknowledgements}

This work was financially supported by National Natural Science Foundation of China Youth Science Foundation ( No.11501499) , Zhaoqing science and technology plan projects ( No.2013K008 ) and by Guangdong province science and technology plan projects ( No. 20110102 ) .

\section{References}

[1]. C.Hindman,K.B.Ousterhout.Developing a flexible sheet forming system[J].J.Materials Processing Technology,2000,99(7):38-48.

[2]. Webb,R.D.,D.E.Hardt.A transfer function description of sheet metal forming for process control[J].Trans.ASME,J.Engg.Industry,1991,133(2):44-52.

[3]. FU Ze-min, GONG Pan, CHEN Wei. Performance analysis of closed-loop control system based on incremental air-bending forming of sheet metal. Material Science \& Technology. Vol .21 (2013) No.3, p. 137-142.

[4]. FU Z M, MO J H. Multiple-step incremental air-bending forming of high-strength sheet metal based on simulation analysis[J]. Materials and Manufacturing Processes, Vol .25 (2010) No.2, p. 806-816.

[5]. PANG Cun-suo, LIU Lei, SHAN Tao.Time-Frequency Analysis Method Based on Short-Time Fractional Fourier Transform[J]. Acta Electronica Sinica,Vol .42 (2014) No.2, p. 347-351.

[6]. Ran Tao, Yan-Lei Li, Yue Wang. Short-time fractional fourier transform and Its applications [J]. IEEE Trans Signal Process. Vol. 58 (2010) No. 5, p. 2568-2580. 